\title{
BMJ Open Characterising the research profile of the critical care physiotherapy workforce and engagement with critical care research: a UK national survey
}

\author{
Bronwen Connolly, ${ }^{1,2,3,4}$ Laura Allum, ${ }^{1}$ Michelle Shaw, ${ }^{5}$ Natalie Pattison, ${ }^{6}$ Paul Dark ${ }^{7}$
}

To cite: Connolly B, Allum L, Shaw M, et al. Characterising the research profile of the critical care physiotherapy workforce and engagement with critical care research: a UK national survey. BMJ Open 2018;8:e020350. doi:10.1136/ bmjopen-2017-020350

- Prepublication history and additional material for this paper are available online. To view these files, please visit the journal online (http://dx.doi. org/10.1136/bmjopen-2017020350).

Received 29 0ctober 2017 Revised 13 April 2018 Accepted 20 April 2018
Check for updates

For numbered affiliations see end of article.

Correspondence to

Bronwen Connolly;

bronwen.connolly@nhs.net

\section{ABSTRACT}

Objective To characterise the research profile of UK critical care physiotherapists including experience, training needs, and barriers and enablers to engagement in critical care research. 'Research' was defined broadly to encompass activities related to quantitative and qualitative studies, service evaluations, clinical audit and quality improvements.

Design Closed-question online survey, with optional freetext responses.

Setting UK critical care community.

Participants UK critical care physiotherapists, regardless of clinical grade or existing research experience.

Results 268 eligible survey responses were received during the 12-week study period (21 incomplete, 7.8\%). Respondents were based in university-affiliated $(n=133$, 49.6\%) and district general $(n=111,41.4 \%)$ hospitals, and generally of senior clinical grade. Nearly two-thirds had postgraduate qualifications at master's level or above $(n=163,60.8 \%)$. Seven had a doctoral-level qualification. Respondents reported a range of research experience, predominantly data acquisition $(n=144,53.7 \%)$ and protocol development $(n=119,44.4 \%)$. Perceived research training needs were prevalent, including topics of research methods, critical literature appraisal, protocol development and statistical analysis (each reported by $\geq 50 \%$ respondents). Multiple formats for delivery of future research training were identified. Major barriers to research engagement included lack of protected time $(n=220,82.1 \%)$, funding $(n=177,66.0 \%)$ and perceived experience $(n=151,56.3 \%)$. Barriers were conceptually categorised into capability, opportunity and motivation themes. Key enabling strategies centred on greater information provision about clinical research opportunities, access to research training, secondment roles and professional networks.

Conclusions UK critical care physiotherapists are skilled, experienced and motivated to participate in research, including pursuing defined academic research pathways. Nonetheless wide-ranging training needs and notable barriers preclude further involvement. Strategies to harness the unique skills of this profession to enhance the quality, quantity and scope of critical care research, benefiting from a multiprofessional National Clinical Research Network, are required.

\section{Strengths and limitations of this study}

- Critical care is a complex specialty by nature requiring a multiprofessional and research-enabled workforce to maximise research planning and delivery for optimum patient benefit.

- This is the first survey to detail the research profile of UK critical care physiotherapists to assist in building research capacity within the critical care workforce.

- Barriers and enablers to engagement of critical care physiotherapists in research have been identified to support development of strategies to enhance future involvement.

- Strengths of study methods include sustained use of multiple and diverse routes of survey dissemination, ease and speed of online completion, and potential for replicability.

- Potential limitations include a profession-specific target population, lack of known denominator to determine accurate response level and predominance of clinician respondents over those from academic institutions.

\section{INTRODUCTION}

Critical care is an identified specialty therapy arena within the UK's National Institute for Health Research (NIHR) (https://www. nihr.ac.uk/nihr-in-your-area/critical-care/). National and local Clinical Research Network (CRN) infrastructure is synchronised to provide oversight and logistical support enabling high-quality conduct and delivery of the NIHR research portfolio (https://www. nihr.ac.uk/research-and-impact/nihr-clinical-research-network-portfolio/-portfolio/). Currently, $96 \%$ of National Health Service (NHS) England's intensive care units (ICUs) contribute to clinical research studies, one of the most engaged critical care research networks internationally. ${ }^{1}$ The NIHR Critical Care National Specialty Group (CC NSG) comprises local CRN representatives who coordinate and review the national critical 
care clinical research portfolio. Since 2014 the CC NSG, currently led by physicians, has engaged physiotherapy representation within it. This is alongside other allied health professions (AHPs), including nursing and pharmacy, recognising that physiotherapists are key members of the multiprofessional team contributing to achievement of its research agenda. Fostering multiprofessional research workforce development and sustainability is an important mission for the CC NSG.

Developing a strategic approach to research capacity building in AHPs, such as physiotherapy, may be complex but is important for enhancing healthcare research across basic science, translational, service delivery and implementation. ${ }^{2}$ Impact and value are seen at individual, team and clinical service levels when research roles for AHP are invested in. ${ }^{3}$ Furthermore, increasing emphasis on evidence-based practice has required greater potential and expectation for AHP assimilation of research to inform the clinical decision-making process. ${ }^{45}$ However, contributing to multiprofessional research effort at scale in NHS critical care could require involvement of clinicians trained to appropriate regulatory Good Clinical Practice (GCP) standards, ${ }^{6}$ but who lack other formal research training or experience. ${ }^{7}$ Identifying researchtrained physiotherapists and establishing levels of skill, competency and expertise would contribute significantly to supporting the planning and delivery of the best research for patient benefit. In addition, this would facilitate and build collaborations, and raise recognition of the profession.

Intensive care medicine, as a profession, encourages exposure to clinical research at varying stages of professional training for all trainee clinicians and a smaller number choose a more structured clinical academic research training pathway. ${ }^{8}$ In the absence of a formal postgraduate clinical training programme for critical care physiotherapy, clinicians have empirically integrated research commitments within existing clinical roles to acquire skills and experience, supplemented by postgraduate qualifications although access to these may be subject to local variability in financial and logistical support. The advent of the NIHR over the last decade has provided dedicated AHP pathways for pursuing a defined clinical academic career (https://www.nihr.ac.uk/funding-andsupport/funding-for-training-and-career-development/ training-programmes/). However, capturing these data at a profession-wide level is challenging with a paucity of work in this field. This survey, therefore, aimed to characterise the research profile of UK critical care physiotherapists, perceived training needs and barriers to engagement in critical care research.

\section{METHODS}

\section{Study design and ethical approval}

This study involved the development of a national online survey of UK critical care physiotherapists conducted on behalf of the UK NIHR CG NSG. The survey did not require ethical approval (UK Health Research Authority ${ }^{9}$ ), and no local institutional research and development department approval was necessary (Guy's and St. Thomas' NHS Foundation Trust, London, UK). Participation was voluntary and consent confirmed by accessing and completing the online survey. Inclusion of participant name was optional, known only to two of the researchers (BC, LA) and used only to facilitate follow-up of incomplete surveys. This study was conducted and reported in keeping with suggested good practice for surveys ${ }^{10}$ and adhering to the Checklist for Reporting Results of Internet E-Surveys. ${ }^{11}$

\section{Sampling and survey population}

Survey respondents included any UK critical care physiotherapist, regardless of existing research experience or clinical grade and employed in adult or paediatric subspecialties. At present, there is no formal database of such clinicians, and we adopted a pragmatic approach to enable responses from as many clinicians as possible within a defined time frame who aligned themselves as a physiotherapist specialising in critical care. Responses from non-physiotherapists were excluded.

\section{Survey development}

An online survey was designed by the authors (online supplementary, section E1). The survey was modelled and expanded on a recently published survey of intensive care medicine trainees with a similar aim, ${ }^{7}$ with additional content considered relevant by the authors for addressing the current study aims. Item generation and reduction were completed internally by the review authors during survey design which also allowed for confirmation of both content and construct validity. ${ }^{12}$ The draft survey was developed offline; clinical sensibility and face validity testing were conducted through piloting the survey with six critical care physiotherapists of varying clinical seniority grade and existing research experience to refine the quality and interpretation of questions. ${ }^{13}$ Feedback was requested on (1) comprehension and interpretation of questions, (2) flow, salience, acceptability and ease of completion, (3) identification of missing items requiring questioning or response options to existing questions and (4) time required for survey completion. ${ }^{12}$ Comments from this process resulted in clarification of seven questions and additional responses added to six questions. On average, the survey was reported not to take more than $10 \mathrm{~min}$ to complete. Once transferred to the online electronic format, the final survey was tested by one further independent physiotherapist.

The final survey consisted of four sections: (1) background (demographics), (2) research experience, (3) research training options and (4) barriers to research engagement. 'Research' encompassed activities related to quantitative studies, qualitative studies, service evaluations, clinical audit and quality improvements. If completed in full, the survey totalled 25 questions. The majority of questions were closed-question nominal format involving 
response selection from multiple non-ranked options. Where applicable, questions always contained an 'other' option to enable free-text comments from respondents. Question layout followed recommended approaches, for example, categorised into subsections, numbered and with response options appearing on separate lines, with multiple item screens and short-entry boxes. ${ }^{12}$ Furthermore, electronic functionality to enhance completion was optimised, for example, filtering of questions according to 'yes/no' response, and limiting progression until specific answers provided.

\section{Survey distribution}

Survey distribution occurred for an a priori defined 12-week period, with an additional 4-week follow-up period to contact respondents with missing data. A personalised opening cover letter was included and the lead author's contact details were circulated on the survey link to respond to any individual queries regarding survey completion. An electronic link enabled respondents to access the online survey and a variety of strategies to access potential respondents were employed including: the NIHR CC NSG and CRN distribution lists (members of the NIHR CC NSG, and representatives of all NHS Trusts within each of the local CRN areas respectively; individuals on these lists were requested to disseminate the survey to their local critical care physiotherapy colleagues); advertising on the NIHR CC NSG website; the UK Critical Care Research Group distribution list (any clinician who has attended the national meeting of this group in the last 3 years); clinical professional specialist interest group websites and membership newsletters; local networks of clinical colleagues; social media links; a national UK critical care course for physiotherapists and snowballing. Regular recirculation of the survey link was conducted via these routes during the 12-week period. At the end, incomplete survey responses were identified. Where respondents left contact details attempts were made to request missing data. Response data were recorded electronically in bespoke survey software (Survey Monkey, San Mateo, California, USA, https://www.surveymonkey.net/) and then exported into Microsoft Excel format (Microsoft Office 2013, Microsoft, Redmond, Washington, USA) for analysis.

\section{Patient and public involvement}

No patients or public were involved in any aspect of this study as it focused on development of a clinician-targeted survey to determine research engagement.

\section{Data analysis}

Quantitative data were analysed descriptively using counts and percentages. Ordinal data were reported as medians (IQR). Qualitative comments were analysed for recurrent themes using free-text analysis ${ }^{14}$ by one researcher (LA) and reviewed by the primary author (BC), removing any potentially identifiable text in advance. One researcher (BC) additionally mapped results to a behavioural change framework involving three essential interacting conditions (capability, opportunity, motivation) that modify and influence the behaviour; the COM-B system. ${ }^{15}$ In this study, the 'behaviour' was critical care physiotherapy research engagement, and findings were best placed under the subheadings of each condition (Capabilityphysical and psychological; Opportunity-social and physical; Motivation-automatic and reflective). In the absence of a known denominator for the total number of UK critical care physiotherapists, it was not possible to set a target a priori response rate. Instead, the overall number of responses acquired during the 12-week survey period were collated. Analyses were performed using GraphPad Prism V.7.0d (GraphPad Software, La Jolla, California, USA, www.graphpad.com).

\section{RESULTS}

\section{Respondents}

Two hundred and seventy-one responses were received during the survey period (30 August to 22 November 2016, with additional follow-up as aforementioned), of which three were excluded ( $\mathrm{n}=2$ duplicate, $\mathrm{n}=1$ non-physiotherapist), leaving 268 in the final analysed sample. Twenty-one responses remained incomplete after attempted contact $(7.8 \% \quad(\mathrm{n}=16 \mathrm{no}$ contact details $))$. Demographic data for the cohort are provided in table 1. Respondents were almost equally based within university-affiliated $(\mathrm{n}=133,49.6 \%)$ or district general $(\mathrm{n}=111,41.4 \%)$ hospitals, although the vast majority were located in England ( $n=234,87.3 \%)$. Median (IQR) clinical seniority of respondents was Band 7 (6-7) indicating a senior, specialist grade (higher bandings indicate greater seniority). Nearly two-thirds of respondents had a postgraduate qualification at master's level or above (including individual modules) $(\mathrm{n}=163,60.8 \%)$, with funding provided locally in half of cases $(n=132,49.3 \%)$. Seven respondents had a doctoral level qualification.

\section{Research experience}

Two respondents did not complete this section $(0.7 \%)$. Of the remaining 266 respondents, $227(84.7 \%)$ indicated existing research experience. Frequency of involvement in types of research activity varied (median (IQR) 2 (1-3) different activities). Most commonly respondents indicated experience of participation in local/regional audits or service evaluations ( $\mathrm{n}=204)$, with 116 respondents reporting attending a research-related course. One hundred and twenty-nine respondents reported involvement in either multisite or single-site studies, and 76 had research publication experience (first, coauthor or senior author). Ninety-one respondents had submitted a conference abstract, and 31 had completed a dedicated postgraduate research qualification.

Sixty-five respondents (24.2\%) indicated current involvement in research as chief $(n=13)$, principal $(n=17)$ or coinvestigator $(n=21)$ or in a research physiotherapist/assistant role $(n=21)$. Other roles included 


\begin{tabular}{|c|c|}
\hline Characteristic & Respondents $(n=268)$ \\
\hline \multicolumn{2}{|l|}{ Employment organisation } \\
\hline University-affiliated hospital & $133(49.6)$ \\
\hline District general hospital & $111(41.4)$ \\
\hline Other-specialist centre & $10(3.7)$ \\
\hline Unclassified & $8(3.0)$ \\
\hline University & $5(1.9)$ \\
\hline Community & $1(0.4)$ \\
\hline \multicolumn{2}{|l|}{ Location } \\
\hline England & $234(87.3)$ \\
\hline Scotland & $16(6.0)$ \\
\hline Wales & $12(4.5)$ \\
\hline Northern Ireland & $6(2.2)$ \\
\hline \multicolumn{2}{|l|}{ Grade of seniority* } \\
\hline 5 & $10(3.7)$ \\
\hline 6 & $73(27.2)$ \\
\hline 7 & $127(47.4)$ \\
\hline $8 a$ & $36(13.4)$ \\
\hline $8 b$ & $4(1.5)$ \\
\hline Other (not specified) & $5(1.9)$ \\
\hline Not given & $13(4.9)$ \\
\hline \multicolumn{2}{|l|}{ Postgraduate qualification $†$} \\
\hline Master of science & $80(29.9)$ \\
\hline Master's level module & $66(24.6)$ \\
\hline PG certificate & $37(13.8)$ \\
\hline PG diploma & $21(7.8)$ \\
\hline Master of research & $10(3.7)$ \\
\hline None & $9(3.4)$ \\
\hline PhD & $7(2.6)$ \\
\hline Professional doctorate & 0 \\
\hline Other $\neq$ & $35(13.1)$ \\
\hline \multicolumn{2}{|l|}{ Funding source $†$} \\
\hline Local organisation & $132(49.3)$ \\
\hline Self-funded & $73(27.2)$ \\
\hline Established funding pathway & $29(10.8)$ \\
\hline Professional body & $15(5.6)$ \\
\hline Other§ & $4(1.5)$ \\
\hline
\end{tabular}

Data reported as $\mathrm{n}(\%)$.

*Indicated UK Agenda for change pay structure for allied health professionals; higher numbers (and consecutive letters) indicate more senior, specialist clinical grades.

tIndicates counts representing frequency of occurrence where multiple options could be selected, and totals will exceed 268 (100\%).

¥'Other' categories include: currently undertaking a postgraduate qualification (MSc, $\mathrm{PhD}$, MRes), $\mathrm{n}=14$; miscellaneous mix, $\mathrm{n}=7$; leadership/education qualification, $n=6$; prescribing qualification, $\mathrm{n}=3$; preregistration $\mathrm{MSc}, \mathrm{n}=3$.

$\S$ 'Other' categories include: charity, $n=3$; Specialist Interest Group, $\mathrm{n}=1$.

strategic positions, for example, as director of an institutional research centre. Respondents were predominantly involved in quantitative study types (randomised
Table 2 Previous or current research activity experience of respondents

\begin{tabular}{ll}
\hline Research activity & Responses* \\
\hline $\begin{array}{l}\text { Data acquisition/completion of } \\
\text { outcomes measures or assessments }\end{array}$ & $144(53.7)$ \\
\hline $\begin{array}{l}\text { Protocol development } \\
\text { Recruitment and consent }\end{array}$ & $119(44.4)$ \\
$\begin{array}{l}\text { Statistical analysis and data } \\
\text { interpretation }\end{array}$ & $81(30.6)$ \\
Intervention delivery & $75(28.0)$ \\
\hline Database management & $69(26.8)$ \\
\hline $\begin{array}{l}\text { Patient and public involvement and } \\
\text { engagement }\end{array}$ & $68(25.4)$ \\
\hline $\begin{array}{l}\text { Ethics/research and development } \\
\text { approvals application process }\end{array}$ & $64(23.9)$ \\
\hline Manuscript preparation/writing & $63(23.5)$ \\
\hline Screening for eligibility & $60(22.4)$ \\
\hline Completion of study case report forms & $46(17.2)$ \\
\hline None & $17(6.3)$ \\
\hline
\end{tabular}

*Date reported as $n(\%): n=268, n=257$ respondents, $n=11$ missing data. Multiple options permitted per respondent.

and non-randomised controlled trials, $\mathrm{n}=22$; observational studies, $n=23$ ), and reported methodological and review-based research (feasibility studies, $n=19$, systematic review, $n=2$, methodological study, $n=1$ ) and qualitative $(n=20)$ research design. Survey $(n=4)$, epidemiological $(n=4)$ and case study, proof-of-concept and mixedmethods ( $\mathrm{n}=1$ each) research studies were also reported. The majority of studies that respondents were involved in were either publicly or self-funded $(n=53,81.5 \%)$.

Two hundred and fifty-seven respondents (95.9\%) described research activities they had previous or current experience of (table 2). More than $50 \%$ had been involved with data acquisition or completion of outcome measure assessment in studies, followed by protocol development. The vast majority of respondents had no allocated time for research in their current role $(n=210,78.4 \%)$. On average, respondents with some allocated research time ranged between 3 days/week and full time $(n=4 \mathrm{~s}, 1.5 \%)$, between 1 and up to 3 days/week $(n=15,5.6 \%)$, less than 1 day/week $(n=18,6.7 \%)$ and less than 1 day/month $(\mathrm{n}=9,3.3 \%)$.

Seventy-one respondents $(26.5 \%)$ had completed GCP training. Of the remaining 186 respondents, 149 (55.6\%) reported a lack of familiarity with what GCP involved, $27(10.1 \%)$ reported they would like to complete GCP training but could not access it locally, 7 (2.6\%) were scheduled to attend and $3(1.1 \%)$ did not feel GCP training was necessary.

\section{Research training needs}

Respondents were asked to identify research training topics they would benefit from (table 3). Two hundred and fifty-one respondents $(93.7 \%)$ completed this 
Table 3 Research training needs of respondents

\begin{tabular}{ll}
\hline Research training need & Responses* \\
\hline Statistical analysis & $132(49.3)$ \\
Research methods & $122(45.5)$ \\
\hline Protocol development & $121(45.1)$ \\
\hline Critical appraisal of literature & $119(44.4)$ \\
\hline Applying for grant funding & $101(37.7)$ \\
Mentorship & $96(35.8)$ \\
\hline Ethics/research and development & $96(35.8)$ \\
application process & \\
\hline Data management & $96(35.8)$ \\
Writing a manuscript for publication & $92(34.3)$ \\
\hline Systematic review/meta-analysis/synthesis & $91(34.0)$ \\
\hline Applying for individual funding & $85(31.7)$ \\
\hline Writing a scientific abstract & $82(30.6)$ \\
\hline Research team collaboration & $69(25.7)$ \\
\hline Recruitment and consent & $45(16.8)$ \\
\hline Epidemiology & $41(15.3)$ \\
\hline
\end{tabular}

*Data reported as $n(\%): n=268, n=223$ respondents, $n=17$ missing data, $\mathrm{n}=28$ reporting no research training required and not categorised.

question. Most frequently reported topics included research methods, critical appraisal of literature, protocol development and statistical analysis, all identified by at least $50 \%$ of respondents. Least reported training topics included epidemiology, and recruitment and consent. Respondents $(n=250,93.3 \%)$ reported a variety of methods of delivery of research training topics including courses/workshops run either on a weekday (full day, $\mathrm{n}=135(50.4 \%)$, half-day, $\mathrm{n}=62(23.1 \%)$ ), weekend (full day, $\mathrm{n}=76(28.4 \%)$, half-day, $\mathrm{n}=37(13.8 \%))$ or evening $(n=33,12.3 \%)$ or via online $(n=145,54.1 \%)$. Analysis of free-text comments from respondents in relation to this question is provided in the online supplementary table E1. Twenty-eight respondents $(10.4 \%)$ reported they would not be interested in any research training.

\section{Barriers and enablers to research engagement}

Of respondents not currently involved in research $(\mathrm{n}=195$, $72.8 \%)$, the vast majority $(n=167,85.6 \%)$ indicated they would like the opportunity. Research activities that respondents expressed interest in included data collection/recording $(\mathrm{n}=142,53.0 \%)$; leadership and conduct of own projects $(\mathrm{n}=129,48.1 \%)$; dissemination activities $(\mathrm{n}=114,42.5 \%)$; data analysis and interpretation $(\mathrm{n}=109$, $40.7 \%)$; writing abstracts for conference submission $(\mathrm{n}=96,35.8 \%)$; manuscript writing for publication $(\mathrm{n}=87$, $32.5 \%)$; recruitment and consenting $(n=83,31.0 \%)$; eligibility screening ( $\mathrm{n}=72$ 260.9\%).

All respondents, regardless of existing involvement in research, were asked to indicate perceived barriers to physiotherapy involvement in critical care research, and initiatives to improve this (termed 'enablers') (table 4).
Table 4 Barriers and enablers to physiotherapy involvement in critical care research

\begin{tabular}{ll}
\hline Barrier & Responses* \\
\hline Lack of protected time & $220(82.1)$ \\
\hline Lack of funding & $177(66.0)$ \\
\hline Lack of experience & $151(56.3)$ \\
\hline $\begin{array}{l}\text { No critical care-related research } \\
\text { conducted in physiotherapy department }\end{array}$ & $110(41.0)$ \\
\hline $\begin{array}{l}\text { Lack of confidence } \\
\text { Insufficient skill set }\end{array}$ & $110(41.0)$ \\
$\begin{array}{l}\text { Unsure what opportunities are available } \\
\text { and/or unsure who to approach to find }\end{array}$ & $96(36.2)$ \\
out & \\
$\begin{array}{l}\text { Lack of support from senior staff/ } \\
\text { management }\end{array}$ & $86(32.1)$ \\
\hline $\begin{array}{l}\text { Insufficient knowledge base } \\
\text { No research currently conducted in } \\
\text { critical care department }\end{array}$ & $69(25.7)$ \\
$\begin{array}{l}\text { Clinical rotations too short to allow } \\
\text { involvement }\end{array}$ & $35(13.1)$ \\
\hline
\end{tabular}

\section{Enabler}

Greater information about local critical 185 (69.0) care physiotherapy studies

Wider advertising of opportunities for $153(57.1)$ involvement

$\begin{array}{ll}\text { Increased access to research training } & 150(56.0) \\ \begin{array}{l}\text { Creation of secondment positions into } \\ \text { research teams }\end{array} & 150(56.0) \\ \begin{array}{l}\text { Greater information about local critical } \\ \text { care studies }\end{array} & 149(55.6)\end{array}$

National physiotherapy network

$147(54.9)$

to link research-active critical care

physiotherapists

Increased engagement at senior staff/ 120 (44.8) management level

Creation of combined clinical-academic 114 (42.5) positions

\begin{tabular}{lc}
$\begin{array}{l}\text { Greater familiarity/understanding of } \\
\text { available funding sources }\end{array}$ & $114(42.5)$ \\
$\begin{array}{l}\text { Greater support from critical care } \\
\text { colleagues, for example, intensivists }\end{array}$ & $102(37.1)$ \\
$\begin{array}{l}\text { Knowledge of key contacts within local } \\
\text { organisation }\end{array}$ & $84(31.3)$ \\
$\begin{array}{l}\text { Option for including as a rotational } \\
\text { objective }\end{array}$ & $66(24.6)$ \\
\hline
\end{tabular}

*Data reported as $n(\%): n=268 ; n=244$ respondents, $n=24$ missing data; enablers, $n=247$ respondents, $n=21$ missing data.

Most frequently identified barriers were lack of protected time $(n=220,82.1 \%)$, lack of funding $(n=177,66.0 \%)$ and lack of experience $(n=151,56.3 \%)$. Key enablers centred on information provision including knowledge of local critical care physiotherapy studies and around 
opportunities for involvement in studies. Analysis of freetext comments from respondents in relation to this question is reported in the online supplementary table E2. New themes of barriers that were identified centred on the profile of research within the physiotherapy profession, and the profile of physiotherapists within the wider research community.

\section{Qualitative data}

Qualitative free-text comments from respondents regarding any aspect of the survey were analysed are summarised (online supplementary tables E3 and E4). In the majority of cases, comments reflected existing response options to previous survey questions, for example, mentorship to assist those commencing involvement in research, lack of protected time, the potential benefits of mentorship and peer support or greater information provision about local critical care research studies. However, these comments were considered valuable for providing an additional personal narrative to contextualise the quantitative data.

\section{DISCUSSION}

This study reports the first findings of their kind detailing characteristics of experience, training and engagement in research of the UK critical care physiotherapy workforce. The results demonstrate a skilled, experienced and motivated workforce constrained by logistical, knowledge-related and professional cultural factors. Key enablers to research engagement primarily centre on improvements in information provision around critical care physiotherapy and non-physiotherapy studies, broadening opportunities for formal research involvement, increased access to training and greater numbers of secondment opportunities into established research groups. Findings from this survey underscore the importance and value of building research capacity in the critical care physiotherapy profession, ${ }^{2}{ }^{16}$ and enable the prioritisation of actions to support developing and sustaining a research-enabled critical care workforce involving physiotherapy.

\section{Significance of the findings}

Research activity in UK ICUs is evidently high, as reflected by the percentage of units supporting the national research portfolio. However, this does not appear commensurate with equally high levels of research involvement by physiotherapists. These survey findings suggest a potential disconnect, highlighted by the depth of detail we have captured at individual clinician level in particular around barriers to involvement, in contrast to the relatively insensitive metrics used to determine research delivery at a unit level. Clinicians indicated a wide range of research experience that, if harnessed and nurtured appropriately, could support future studies led by, and in collaboration with, critical care physiotherapists. In turn, this could assist in maintaining and diversifying the national portfolio beyond existing levels. Critical care is by nature a complex specialty provided by a multiprofessional team; consequently the best research for patient benefit is likely to arise secondary to engagement of all members of that team. These findings also mirror the international evidence base exploring AHP engagement in research ${ }^{47-20}$ supporting their generalisability and confirming consistent themes across disciplines and different healthcare jurisdictions.

To understand factors contributing to current levels of physiotherapy engagement in critical care research, results were broadly mapped to a common behavioural change model, the COM-B framework (figure 1). ${ }^{15}$ Addressing any component of COM-B can facilitate behaviour modification; in this instance, the 'behaviour' being involvement in research. For example, major opportunity-related barriers reported by clinicians were lack of protected time and funding with clinicians attempting to incorporate research opportunity within day-to-day clinical roles. This scenario of research capacity balanced against resource restriction is not uncommon within physiotherapy, ${ }^{21}{ }^{22}$ and may be difficult to immediately rectify with ever-increasing demands on clinical service delivery and competing priorities. That said, one enabler identified by respondents focused on awareness of available funding sources and this could be facilitated by identifying colleagues with knowledge and experience around identifying funding options for guidance. Insufficient knowledge, skills and confidence (capability) were other important barriers. Increased access to research training was a key enabler that could target this aspect. Importantly though, findings from this survey highlighted the need to consider flexible, multimodal and innovative forms of training in terms of content, design, format and delivery. One-third of respondents reported that identifying key contacts with their local organisation would facilitate involvement in research. While this has obvious practical benefits, it further speaks to the broader concept of requiring role models, mentors and leadership (motivation barrier) to set a template and provide guidance.

Quantitative and qualitative findings from this survey suggested a distinction between clinicians who had transitioned into a defined academic path, for example, research-specific master's level qualifications and/ or subsequent doctoral training, and those who had skill and experience (often considerable) and keenness for research involvement but who preferred to remain primarily clinical facing; the concept of clinical academics and academic clinicians. The extent to which this concept truly exists requires further exploration among critical care physiotherapists, but could highlight differing approaches needed to integrate these different roles into the research community. Presence of a positive research culture (both within physiotherapy departments and ICUs), perceived value of research by own and other professions, and the overall research profile of the physiotherapy profession were all factors 


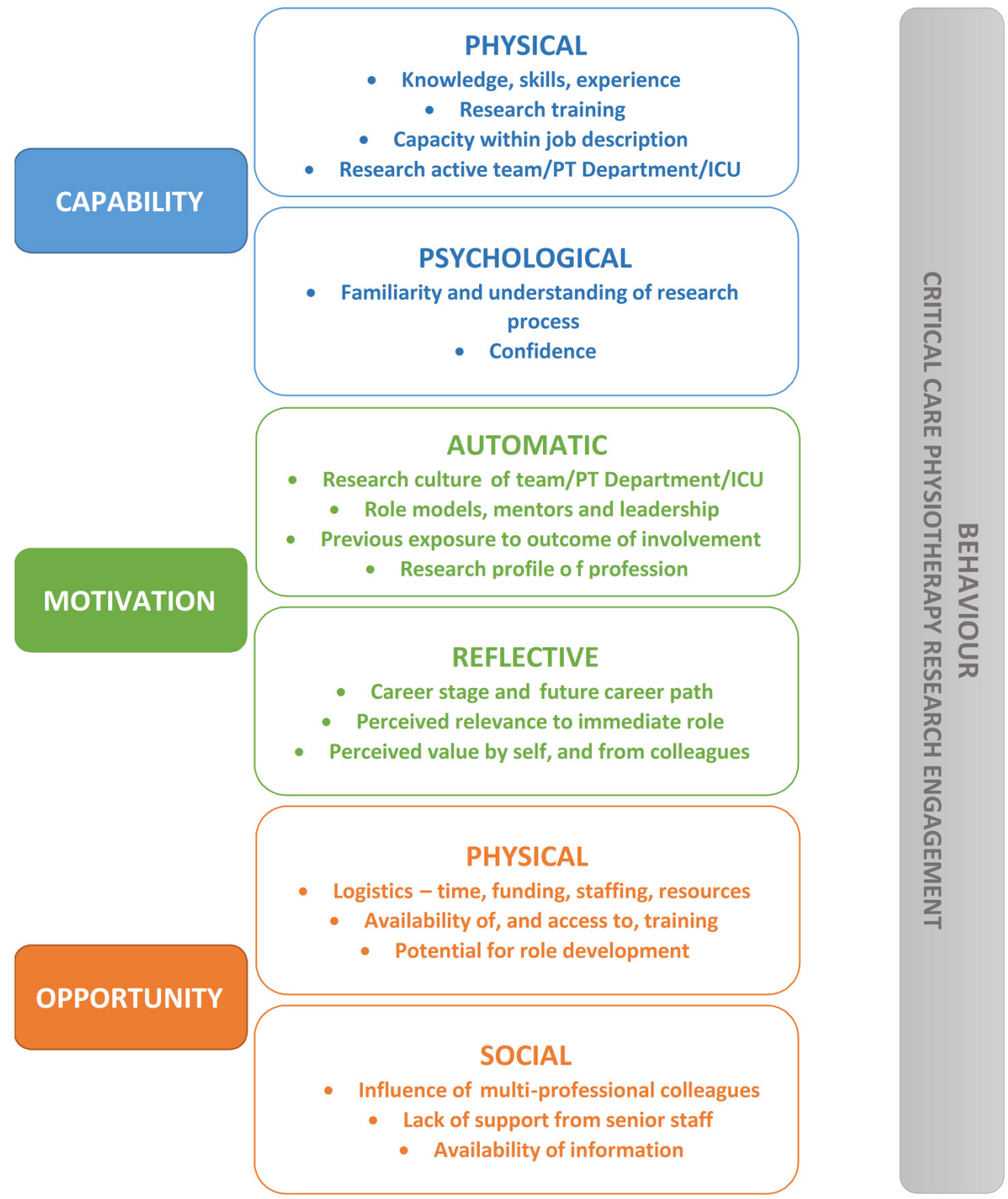

Figure 1 Influencing factors contributing to critical care physiotherapists' involvement in research mapped to the COM-B model. ${ }^{15} \mathrm{COM}-\mathrm{B}$, capability, opportunity, motivation and behaviour; ICU, intensive care unit; PT, physiotherapy.

identified by respondents that influenced their opportunity for involvement in research; in particular recognition from senior management and support from critical care colleagues were reported as beneficial factors. These findings echo similar themes identified from a previous survey of physiotherapy managers' of their departmental staff, ${ }^{21}$ and a separate observational study of physiotherapist researchers having completed PhDs ${ }^{23}$ In this latter study, key suggestions for improving research academic career paths included roles that allowed for clinical research and academic clinical combinations, securing adequate funding for physiotherapy research positions, and enhancing collaboration between academic and clinical researchers. ${ }^{23}$ Certainly fostering partnerships between universities and NHS institutions in the UK, in particular via Academic Health Sciences Centres, for honorary academic appointments could be valuable for accessing academic support and mentorship for clinicians. Furthermore improving patient healthcare through embedding research into routine clinical care is key for the NIHR, in line with NHS constitution for England principles ${ }^{24}-$ this ethos provides support to those aforementioned clinicians wanting greater research exposure while remaining in direct clinical positions.

Moving forwards, these survey findings help to identify strategies to support greater involvement of critical care physiotherapists in research; indeed the impact of these findings could be more wide-reaching in principle relating to other AHPs. Improving information provision around existing studies and secondment opportunities for involvement could be achieved through local and national research-based infrastructure; encouraging links 
between existing professional organisations to combine resources and promote funding and training opportunities; considering alternative models of working to incorporate research time into clinical job descriptions may be required, and engaging managers proactively to recognise the value of research-trained physiotherapists embedded in clinical services; and profiling positive examples of success to increase awareness among the multiprofessional critical care team about the benefit of physiotherapy involvement in studies. In addition, developing peer-support networks akin to that which has been established by intensive care medicine trainees (http://www.raftrainees. $\mathrm{com} /$ ), may be valuable for sharing experiential learning, offering access to mentors and collaborative working, and the newly formed UK AHP/Nursing Network for Critical Care Research is an example of this. Finally, collaborating with other critical care professions to deliver generic research methods training would ensure efficient utilisation of resources and personnel, and likely broaden the depth and breadth of the overall learning experience and foster interprofessional links. Building research skills and training into physiotherapy-specific competencies would mirror the approach taken in other medical specialities. Key to supporting these initiatives is the recent publication of the NIHR CRN Allied Health Professionals Strategy that sets out five strategic goals to realise the potential of AHP contributing to the conduct and delivery of NIHR research across the specialties (available at https:/ /www.nihr.ac.uk/ our-faculty/clinical-research-staff/allied-health-professionals.htm), and significantly highlights this area.

\section{Critique of the method}

These novel data from both quantitative and qualitative analysis highlight the research profile of UK critical care physiotherapists. While the target population of respondents was profession-specific, which could limit generalisability, this study echoes the process and outcomes of a recent survey of UK intensive care medicine trainees which sought to understand how to improve trainee access to critical care research opportunities. ${ }^{7}$ Furthermore, the current survey was in itself non-profession focused, that is, no questions were designed or phrased specifically related to physiotherapy per se. It could therefore easily be replicated across other allied health and nursing roles with little, if any, modification to generate larger volumes of similar characteristic data.

This study benefits from rigorous methods during the development, testing and delivery stages of the survey to optimise data acquisition. The focus of the survey was limited to acquiring descriptive information to provide a baseline phenotype of the current state of critical care physiotherapy engagement in research. Nonetheless further psychometric testing of the survey, for example, formal cognitive interviewing may have been methodologically valuable in its development. ${ }^{25}$ In the future, more detailed and purpose-designed studies to enable deeper exploration of this area may also be valuable, which would facilitate inferential analyses.
A notable strength of this study is the use of sustained, multiple and diverse routes of dissemination for maximising awareness and completion among the target population during the survey period, spanning clinical, research and professional remits. This approach was essential given the absence of a formal central registry for identifying potential respondents. Nonetheless lack of an accurate respondent denominator precludes determining an overall response level, and consequently may challenge the representativeness of findings. Determining the size of the UK critical care physiotherapy workforce is challenging. Health and Care Professions Council (http:// www.hcpc-uk.org/aboutregistration/professions/index. asp?id=11\#profDetails) and NHS Workforce (https:// data.gov.uk/dataset/nhs-workforce-non-medical-staff) data lack specialty-specific granularity to provide meaningful estimates. National critical care staffing recommendations advise at least one whole time-equivalent physiotherapist to every four critical care beds ${ }^{26}$ Using bed capacity data $\left(\mathrm{n}=3536\right.$, as of April 2017, ${ }^{27}$ would suggest a potential sample of 884 UK critical care physiotherapists, although these recommendations are not routinely adopted in practice and therefore this may be an overestimation. To provide some perspective the response level is more than four times the number of physiotherapy members in the UK Intensive Care Society $(\mathrm{n}=60),{ }^{28}$ and is estimated to reflect critical care membership within the Association of Chartered Physiotherapists in Respiratory Care (Personal Communication; 2017 membership 1050, assuming equal distribution across the four core areas of critical care, surgery, long-term conditions and paediatrics (http://www.acprc.org.uk/). Our individual national response levels also closely align with national population levels suggesting the findings reflect the geographical spread of UK critical care physiotherapists (https://www.ons.gov.uk/peoplepopulation andcommunity/populationandmigration/populationestimates/bulletins/annualmidyearpopulationestimates/ latest). However in the future it may be beneficial to explore any potential differences between nations in the context of specific detail of critical care service provision. It is possible that missing data to questions, while overall very low, may have been skewed by the relative proportions of responses which should be considered when interpreting results, for example, defining future training needs. Furthermore, all available opportunities for targeting respondents were adopted, and the achieved response level has provided adequate data to answer the original study aims with consistent themes arising from the data. However, as with all surveys there is potential for inherent self-selection and response bias and there are no formal means of assessing degree of non-response and/ or any differences between characteristics of responders versus non-responders. ${ }^{29} 30$

Choice of online survey completion via electronic link versus alternative routes such as postal, was also pragmatic in light of lack of contact details for potential respondents. A 4-week follow-up period following official 
closure of the survey was included to contact respondents to obtain missing data, a challenge to all survey studies regardless of interface, and within the current sample the overall proportion of missing data was low $(<8 \%)$. This survey primarily targeted clinical rather than academic critical care physiotherapists although responses from these individuals were not specifically excluded but nor were avenues of survey promotion or dissemination via academic organisational routes pursued; ultimately a very small proportion of respondents indicated they were based in university settings. In the future specifically targeting/including academic clinicians may provide valuable information as their experience of engagement in critical care research may differ due to context and environment which may have been missed in the current results. That said, similar challenges around determining an accurate denominator for these individuals may still exist.

Importantly, the definition of a "critical care physiotherapist' was open to individual interpretation to maximise volume and breadth of response level. This was not restricted to any acuity of healthcare setting recognising that research with critically ill patients may transition clinical environments, be irrespective of levels of care, ${ }^{31}$ and indeed continue beyond the acute hospitalisation period. However, it is acknowledged that in the latter stages of recovery physiotherapists from other specialist areas may become involved in delivery of services to postcritical illness patients, and they may not have responded to a survey targeted at 'critical care' physiotherapists. In addition, physiotherapists in both the adult and paediatric sector were eligible to respond and from all clinical grades of seniority to maximise representativeness. Furthermore, a broader definition of the term 'research' was adopted to encompass clinical audit, service evaluations an quality improvements to capture data on all activities that clinicians may be involved in and use broad research-based skills. Again, this approach helps to consider translation of the findings to other non-physiotherapy professions where involvement in this range of activities may occur. Finally, as with all survey data, the findings are relative to the survey period and it is acknowledged that additional numbers of clinicians may have attained postgraduate qualifications or involvement in research in the interim period from survey conduct to publication of results.

\section{CONCLUSION}

UK critical care physiotherapists have skill and experience in many aspects of research. A large number have postgraduate qualifications, including those indicating a defined academic research path. Nonetheless wideranging training needs and notable barriers preclude further involvement. These data may help inform approaches to harness the unique skills of this profession to enhance the quality, quantity and scope of critical care clinical research to maximise patient benefit, within a multiprofessional national CRN and may have international applicability.

\section{Author affiliations}

${ }^{1}$ Lane Fox Clinical Respiratory Physiology Research Centre, Guy's and St.Thomas' NHS Foundation Trust, London, UK

${ }^{2}$ National Institute for Health Research Biomedical Research Centre, Guy's and St. Thomas' NHS Foundation and King's College London, London, UK

${ }^{3}$ Centre for Human and Applied Physiological Sciences, King's College London, London, UK

${ }^{4}$ Department of Physiotherapy, The University of Melbourne, Melbourne, Victoria, Australia

${ }^{5}$ Northern Deanery, Newcastle upon Tyne, UK

${ }^{6}$ School of Health and Social Work, University of Hertfordshire and East and North Hertfordshire NHS Trust, Hertfordshire, UK

${ }^{7}$ Division of Infection, Immunity and Respiratory Medicine, Manchester University NHS Foundation Trust, University of Manchester, Manchester, UK

Acknowledgements The authors would like to gratefully acknowledge the support of Joanne Ashcroft (NIHR Clinical Research Network (CRN) Assistant Specialty Cluster Lead, King's College London) and Tracy Gregg (NIHR CRN Communications Manager) from the National Coordinating Centre, and the NIHR National Clinical Research Network for facilitating the conduct and delivery of this survey. In addition, the authors would like to thank all participants for contributing their time to completion of the survey, and to the clinical and research networks, professional specialist interest groups and individual colleagues for their efforts in circulating the survey throughout the duration of the study period. Data referenced in citation 27 derive from the Case Mix Programme Database. The Case Mix Programme is the national, comparative audit of patient outcomes from adult critical care coordinated by the Intensive Care National Audit and Research Centre (ICNARC). For more information on the representativeness and quality of these data, please contact ICNARC (icnarc@icnarc.org).

Contributors BC conceived the study, developed and conducted the survey, completed data analysis and interpretation and drafted, revised and agreed the final manuscript version for submission. LA contributed to data entry, analysis and interpretation. MS contributed to survey development. NP contributed to study design, survey development and data interpretation. PD contributed to survey development and data interpretation. All authors contributed to manuscript revision and approved the final version for submission. $\mathrm{BC}$ acts as the guarantor for the intellectual integrity of the data.

Funding The authors have not declared a specific grant for this research from any funding agency in the public, commercial or not-for-profit sectors.

Competing interests None declared.

Patient consent Not required.

Provenance and peer review Not commissioned; externally peer reviewed.

Data sharing statement All data pertaining to this study are reported in this manuscript.

Open Access This is an Open Access article distributed in accordance with the Creative Commons Attribution Non Commercial (CC BY-NC 4.0) license, which permits others to distribute, remix, adapt, build upon this work non-commercially, and license their derivative works on different terms, provided the original work is properly cited and the use is non-commercial. See: http://creativecommons.org/ licenses/by-nc/4.0/

(C) Article author(s) (or their employer(s) unless otherwise stated in the text of the article) 2018. All rights reserved. No commercial use is permitted unless otherwise expressly granted.

\section{REFERENCES}

1. National Institute for Health Research Open Data Platform. https:// odpnihracuk/qlikview/ (accessed 28 Oct 2017).

2. Pickstone $\mathrm{C}$, Nancarrow $\mathrm{S}, \mathrm{Cooke} \mathrm{J}$, et al. Building research capacity in the allied health professions. Evidence \& Policy 2008;4:53-68.

3. Wenke RJ, Ward EC, Hickman I, et al. Allied health research positions: a qualitative evaluation of their impact. Health Res Policy Syst 2017;15:6. 
4. Stephens D, Taylor NF, Taylor N, et al. Research experience and research interests of allied health professionals. J Allied Health 2009;38:107E-11.

5 Allied Health Professions into Action: Using Allied Health Professionals to transform health, care and wellbeing. NHS England. 2017 https://www.england.nhs.uk/wp-content/uploads/2017/01/ahpaction-transform-hlth.pdf

6. National Institute for Health Research Good Clinical Practice. https:// wwwnihracuk/our-faculty/clinical-research-staff/learning-anddevelopment/national-directory/good-clinical-practice/ (accessed 28 Oct 2017).

7. Shaw M, Harris B, Bonner S. The research needs of an ICM trainee: The RAFT national survey results and initiatives to improve trainee research opportunities. J Intensive Care Soc 2017;18:98-105.

8 Faculty of Intensive Care Medicine. Guidance on Academic Training in Intensive Care Medicine. 2013 https://www.ficm.ac.uk/./academic training_in_intensive_care_medicine_v_1.0_jan_2013.pdf (accessed 01 Aug 2017).

9. National Health Service, Health Research Authority. Studies involving the NHS in England. http://www.hra.nhs.uk/research-community/ before-you-apply/determine-whether-your-study-is-research/ (accessed 08 Mar 2016)

10. Kelley K, Clark B, Brown V, et al. Good practice in the conduct and reporting of survey research. Int J Qual Health Care 2003;15:261-6.

11. Eysenbach G. Improving the quality of Web surveys: the Checklist for Reporting Results of Internet E-Surveys (CHERRIES). J Med Internet Res 2004;6:e34.

12. Burns KE, Duffett $M$, Kho ME, et al. A guide for the design and conduct of self-administered surveys of clinicians. CMAJ 2008;179:245-52.

13. Beatty PC, Willis GB. Research synthesis: the practice of cognitive interviewing. Public Opin Q 2007;71:287-311.

14. Hsieh HF, Shannon SE. Three approaches to qualitative content analysis. Qual Health Res 2005;15:1277-88.

15. Michie S, van Stralen MM, West R. The behaviour change wheel: a new method for characterising and designing behaviour change interventions. Implement Sci 2011;6:42.

16. Janssen J, Hale L, Mirfin-Veitch B, et al. Building the research capacity of clinical physical therapists using a participatory action research approach. Phys Ther 2013;93:923-34.

17. Finch E, Cornwell $P$, Ward EC, et al. Factors influencing research engagement: research interest, confidence and experience in an
Australian speech-language pathology workforce. BMC Health Serv Res 2013;13:144.

18. Roxburgh M. An exploration of factors which constrain nurses from research participation. J Clin Nurs 2006;15:535-45.

19. Pager S, Holden L, Golenko X. Motivators, enablers, and barriers to building allied health research capacity. J Multidiscip Healthc 2012;5:53-9.

20. Williams CM, Lazzarini PA. The research capacity and culture of Australian podiatrists. J Foot Ankle Res 2015;8:11.

21. Skinner EH, Hough J, Wang YT, et al. Physiotherapy departments in Australian tertiary hospitals regularly participate in and disseminate research results despite a lack of allocated staff: a prospective crosssectional survey. Physiother Theory Pract 2015;31:200-6.

22. Janssen J, Hale L, Mirfin-Veitch B, et al. Perceptions of physiotherapists towards research: a mixed methods study. Physiotherapy 2016;102-210-6.

23. Bernhardt J, Tang LS. More options and better job security required in career paths of physiotherapist researchers: an observational study. Aust J Physiother 2008;54:135-40.

24. The NHS Constitution for England. The NHS Constitution and guidance on how to make a complaint about NHS services. https:// www.gov.uk/government/publications/the-nhs-constitution-forengland (accessed 28 Oct 2017).

25. Smith OM, Dale C, Mehta S, et al. Nurse Research Experiences and Attitudes Toward the Conduct of Intensive Care Research: A Questionnaire Study. Crit Care Med 2016;44:153-61.

26. Intensive Care Society. Core Standards for Intensive Care Units. 2013 http://www.ics.ac.uk/ICS/Education/Guidelines_Standards/ ICS/guidelines-and-standards.aspx?hkey=4ed20a1c-1ff8-46e0b48e-732f1f4a90e2 (accessed 14 Dec 2014).

27. Intensive Care National Audit and Research Centre Report. Number of beds recorded within all units participating in the Case Mix Programme 1 September 2016 and 1 April 2017. 2017 https:// wwwicnarcorg

28. Membership Services. Intensive Care Society Report. 2017 http:// www.ics.ac.uk/

29. Burkell J. The dilemma of survey nonresponse. Libr Inf Sci Res 2003;25:239-63.

30. Rubenfeld GD. Surveys: an introduction. Respir Care 2004;49:1181-5.

31. Department of Health. Comprehensive critical care: a review of adult critical care services. London: Department of Health, 2000. 